\title{
Erratum
}

\section{Dimethyl Carbonate (DMC): A Versatile and Environmentally Benign Building Block}

Sunay V. Chankeshwara* Synlett 2008, 624.

This spotlight was inadvertently given No. 228. The correct labelling is Spotlight 230. 